\title{
Feature Extraction of Video Data for Automatic Visual Tool Tracking in Robot Assisted Surgery
}

\author{
J Huang \\ ZJUI, Zhejiang University \\ 718 East Haizhou Road, \\ Haining, Zhejiang 314400 \\ People's Republic of China \\ jinghanhuang@intl.zju.e \\ du.cn
}

\author{
T. Kesavadas \\ ISE, University of Illinois at \\ Urbana-Champaign. \\ 104 S. Mathews Ave. \\ Urbana, IL 61801-3080 \\ kesh@illinois.edu
}

\author{
L Yang \\ ZJUI, Zhejiang University \\ 718 East Haizhou Road, \\ Haining, Zhejiang 314400 \\ People's Republic of China \\ liangjingyang@intl.zju.e \\ du.cn
}

\begin{abstract}
The strong interest in data-driven surgical procedures has motivated the need for automatically analyzing instrument trajectories during surgical procedures to improve the efficacy of modern robot-assisted surgeries. We proposed a single view camera-based approach for automatic video analysis of surgical instruments during robot-assisted minimally invasive procedures. This video data-driven approach uses feature extraction for visual tracking of the surgical instrument, coupled with the kinematics of the pivoted motion in minimally invasive surgery for tool trajectory analysis. The self-contained nature of the approach enables the acquisition of trajectory information without any prior placement of markers or post storage of robot joint trajectories information. Based on visual inspection of the 2D localization results in the image frames, the proposed method demonstrated reasonable recovery of the $3 \mathrm{D}$ positional information of the surgical tools. Driven by the increasingly sophisticated interventional procedures of modern surgeries, this proposed trajectory tracking method provides a means for leveraging the wealth of surgical video data for data analytics in Surgical Data Science. The long-term goal of the study is to contribute towards the advancement of Surgical Data Science through the establishment of a video-based data acquisition technique.
\end{abstract}

\section{CCS Concepts}

- Applied computing $\rightarrow$ Health care information systems • Information systems $\rightarrow$ Process control systems

\section{Keywords}

Visual Tracking; Feature Extraction; Surgical Data Science

\section{INTRODUCTION}

Modern technology has revolutionized the field of surgery providing surgeons with unprecedented precision, dexterity and visual capabilities to perform medical procedures that were impossible in the past. A prominent example is robot-assisted surgery [1], which involves the use of teleoperated slave-

\footnotetext{
Permission to make digital or hard copies of all or part of this work for personal or classroom use is granted without fee provided that copies are not made or distributed for profit or commercial advantage and that copies bear this notice and the full citation on the first page. To copy otherwise, or republish, to post on servers or to redistribute to lists, requires prior specific permission and/or a fee.

ICRCA 2019, July 26-28, 2019, Guangzhou, China

(C) 2019 Copyright is held by the owner/author(s).

ACM ISBN 978-1-4503-7183-4/19/07.

DOI: https://doi.org/10.1145/3351180.3351185
}

manipulator system to perform operation on the patient. To remotely control the slave-manipulator, the surgeon operates on a console with $3 \mathrm{D}$ visual display of the camera view on the operated scene. Coupled with the increasingly growing interest in postoperation quality-of-life [2, 3], there is a paradigm shift in the operation mode from open to minimally invasive procedures where sophisticated imaging and robot technology are extensively adopted [4-7].

While the contribution of robotics and digital technology gained recognition in the field of surgery and equipping surgeons with unprecedented information augmentation and dexterity, modern procedures are becoming increasingly more challenging due to the complexity of procedures. This phenomenon poses both an opportunity for enhanced treatment outcomes as well as challenges to traditional manual competence. To bridge the gap between contemporary digital technology and the highly manual approach, there is a need to tap on the wealth of digital data. This work is motivated by the strong interest in Surgical Data Science $[8,9]$ that aims to objectify existing practice including the process of surgical proficiency training [10-14] and assessment [15-17]. With an increasing reliance on data-driven approaches in medical interventional strategies, there is a need for acquiring and analyzing of the instrument trajectory data during interventional procedures.

To address the aforementioned needs and gap, this work proposes a single view camera-based approach for acquiring trajectory data of surgical instruments during robot-assisted minimally invasive procedures, hence leveraging the wealth of digital video data available. A visual feature detection approach, coupled with the kinematics model of the pivoted motion in minimally invasive surgery, is introduced in this paper. In contract to traditional surgical video analysis, which is usually carried out manually, this self-contained and robust tracking approach provides the means for tapping into the massive pool of data with better ubiquity and operation efficiency.

Unlike existing trajectory tracking methods [18-21], the selfcontained nature of our proposed approach is intended to provide a means for the acquisition of the trajectory data from existing video database of robot-assisted minimally invasive procedures without complicated pre-operative setup, intra-operative placement of artificial markers, and post-operative storage and registration of sensor data in multiple domains. As this method relies solely on video data to perform tracking of the surgical tool and estimation of its pose, there is no need for prior marker placement in the surgical scene and post storage of joint trajectories from the surgical robot. The self-contained nature of 
our approach is intended to provide a means for the acquisition of trajectory information for existing video data.

In the next section, we give an overview of the proposed method discuss the image processing technique used in the method for visual tracking of the surgical tools. The results of our method on a video data acquired during a robot-assisted surgery will be presented in Section III followed by a discussion of the observation in Section IV. Section V concludes this paper by summarizing the contributions and their significances including a brief comment of prospective development of this study.

\section{METHOD}

\subsection{Overview}

The heart of our proposed method consists of a visual tracking technique that uses only information from the natural scene for motion estimation of the tool trajectory. Our work focuses on extracting the planar features that could be used for the solving of the $3 \mathrm{D}$ position of the surgical tool's rigid distal end. This is done without any external sensor except for the very endoscope used for the procedure. Orientation of the articular end effector of the surgical tools is not investigated in this study as this could be solve by existing 2D-3D registration method given the ComputerAided-Design (CAD) model of the specific tool [22, 23]. This 3D model-based registration approach has been demonstrated in a previous study for oral and maxillofacial surgical applications [24, 25]. The technical feasibility of such an approach was further demonstrated with our previous application on real-time augmented reality overlay of 3D model [26]. In this current work however, we will focus on the development and investigation of the feature extraction and visual tracking tasks in the context of robot-assisted surgeries

Figure. 1 illustrates the intended workflow for digitizing the surgical tool trajectory from endoscopic camera- acquired surgical scene. For a given sequential image frames of the surgical tool motion from the video data, we are able to recover the 3D path of the tool shaft through the proposed workflow starting from feature extraction of the images to generating the trajectory profiles of the motion. Image processing of the video sequence in a frame-byframe fashion is performed to extract low level features representing the moving tools. Motion estimation will be performed and eventually represented as the tool motion trajectory for task segmentation and analysis.

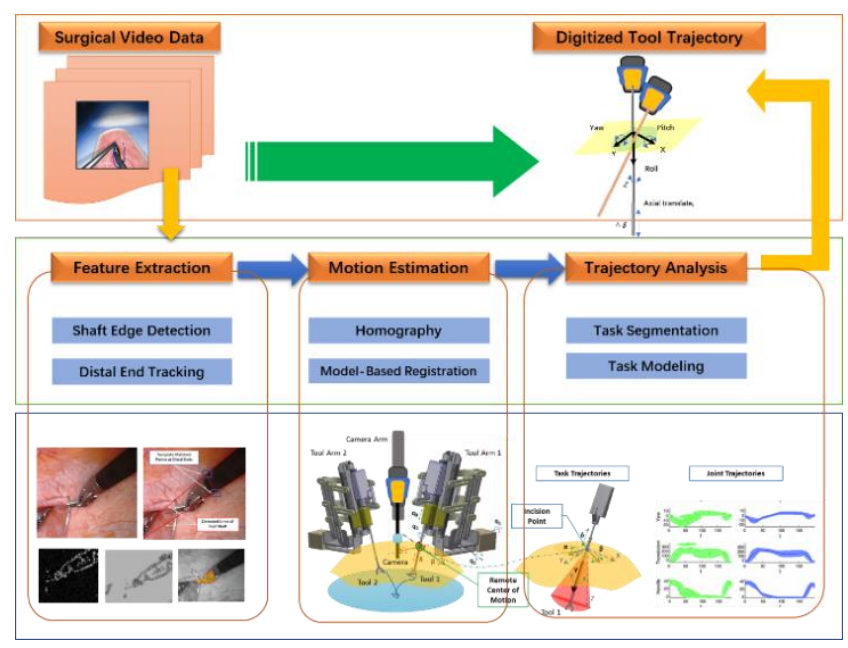

Figure 1. Workflow of Proposed Method.

This paper will focus on the feature extraction method and how tracking failure could be detected effectively using a projective geometry constraint derived from kinematics of the minimally invasive procedures. The developed visual tracking approach lay a foundation for surgical task modeling and analysis through video data analysis of robot-assisted minimally invasive procedures.

\subsection{Feature Extraction}

The first step for acquiring trajectory data from existing surgical video is feature extraction. This is a series of image processing techniques that take in video data and produce extracted features as illustrated in Figure. 2.
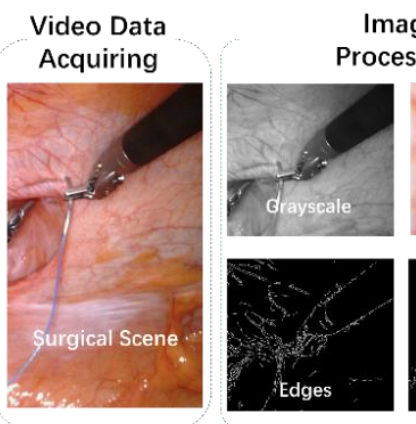

Image

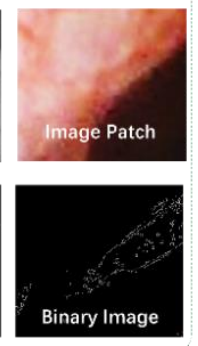

Figure 2. Image Processing Steps to Extract Features from Surgical Scene Acquired using Endoscopic Camera.

\subsubsection{Detection of Tool Shaft: Hough Transformation}

The edge of the tool shaft is detected using linear Hough Transformation [27]. The Cartesian coordinates of potential feature points extracted using Canny Edge detection [28] is mapped to their respective loci in the Hough space (in polar coordinates). This is a mapping of the parameters $(m, c)$ from the linear equation

$$
a x+b y+c=0,
$$

where $m=-a / b$ and $k=-c / b$, to the parameters $(r, \theta)$ in the Hough space expressed as

$$
r=x \cos \theta+y \sin \theta,
$$

As illustrated in Figure 3, the extent of overlapping counts on the coinciding loci determines the parameters of potential line features.

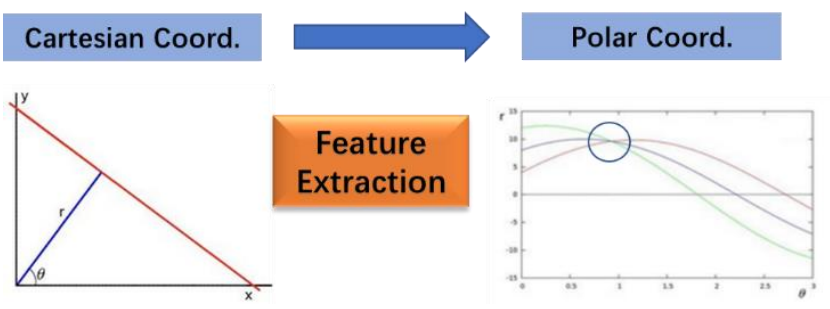

Figure 3. Working Principle of the Hough Transform: Transforming Features from Cartesian to Polar Coordinate System.

A sinusoidal locus can be formed by plotting all the possible values of $(r, \theta)$ associated with a particular feature point at pixel coordinates $(i, j)$ as a single point will produce infinite possible line represented by all points along the continuous sinusoidal 
curve. By further discretizing the Hough space with voxels, the number of counts they coincide with a locus indicates the vote from the potential feature points on a particular Hough space coordinates i.e. line's parameters. Fig. 4 shows the visualization of the all candidate points plotted as the associating locus in the Hough space. The brightest spot (highest voted candidate) of the color coded visualization representing maximum overlaps of locus determines the polar coordinates of the associated line. This associate line is the highest probable line in the scene.

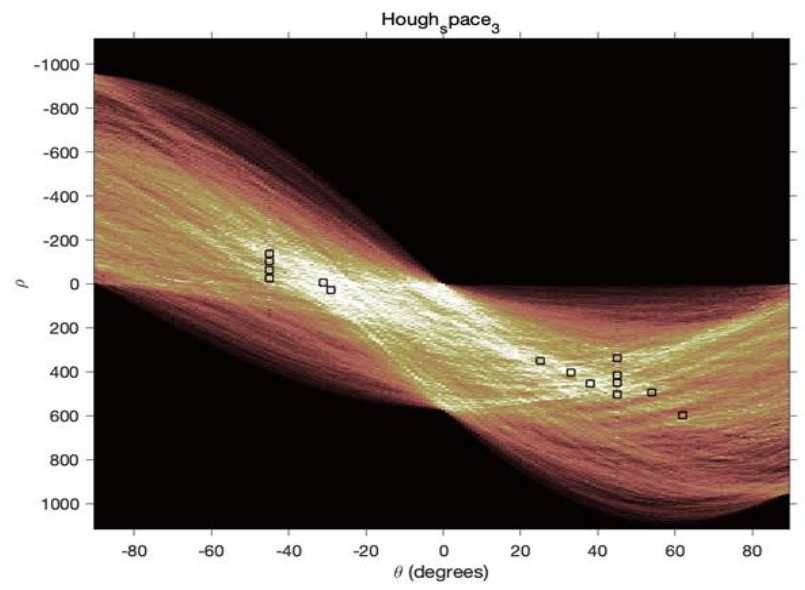

Figure 4. Visualization of all candidate points plotted as the associating locus brightest spot of the color coded visualization represents maximum overlaps of locus determining the polar coordinates of the associated line.

\subsubsection{Tracking of Tool Distal End: Template Match}

The distal end of the surgical instruments is tracked by matching a specific template base over a sequence of image frames. The cross-correlation $w_{c c}(u, v)$ at image coordinates $(u, v)$ of an image patch which is the base template $g(p, q)$ and an image $f(p, q)$ in a particular frame is expressed as

$$
w_{c c}(u, v)=\sum_{p=0}^{P} \sum_{q=0}^{Q} g(p, q) f(p+u, q+v)
$$

for a $\mathrm{P} x \mathrm{Q}$ patch and $\mathrm{U} \times \mathrm{V}$ image. To account for intensity variation, we normalize $w_{c c}(u, v)$ to obtain the coefficient

$$
w_{n c c}(u, v)=\left(\sum_{p=0}^{P} \sum_{q=0}^{Q}\langle G\rangle\langle F\rangle\right) /\left[\left(\sum_{p=0}^{P} \sum_{q=0}^{Q}\langle G\rangle^{2}\right)\left(\sum_{p=0}^{P} \sum_{q=0}^{Q}\langle F\rangle^{2}\right)\right]^{0.5},
$$

where $\langle G\rangle=(g(p, q)-\bar{g})$ and $\langle F\rangle=f(p+u, q+v)-\bar{f}(u, v)$.

Notation $\bar{g}$ and $\bar{f}$ represent the mean intensity value in the template and the window overlapping the patch, respectively. By obtaining the maximum similarity score of the ROI, we can localize the identified matching patch in an image frame of the video sequence.

\subsection{Kinematic Constraint for Failure Detect}

Prior knowledge of the kinematic constraint is useful in formulating effective conditions for outlier removal or failure detection. This approach is common in filter-based techniques, for instance, the use of the Extended Kalman filter [29-31] or more event-based approaches specific to navigation and localization [32-34] . Following the classical method described by Zhang [35], the Intrinsic Parameters, $\mathbf{M}$ and Extrinsic Parameters, $\mathbf{T}$ constitute the camera matrix, which essentially describes the pose of the tracked object with respect to the camera coordinate plane Computing this information every frame will provide us with trajectory information of the surgical tools.

The motion of the surgical instruments can be expressed in the form of a homogenous transformation matrix as

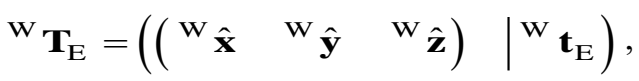

where the first three columns of unit vectors on the left represent the orthogonal axes of the rotational matrix. The last column is the position vector of the tool's end-effector with an assigned reference frame denoted by $\{\mathrm{E}\}$ in world coordinate system $\{\mathrm{W}\}$. Figure 5 illustrates the kinematic constraint of the operation due to the incision point of the tool. This kinematics constraint at the incision point determines the remote-center-of-motion (RCM) [36, 37]for the robot manipulator limiting the surgical tool motion to a 4-DOF task space, excluding the wrist and open-close motion of the end-effector

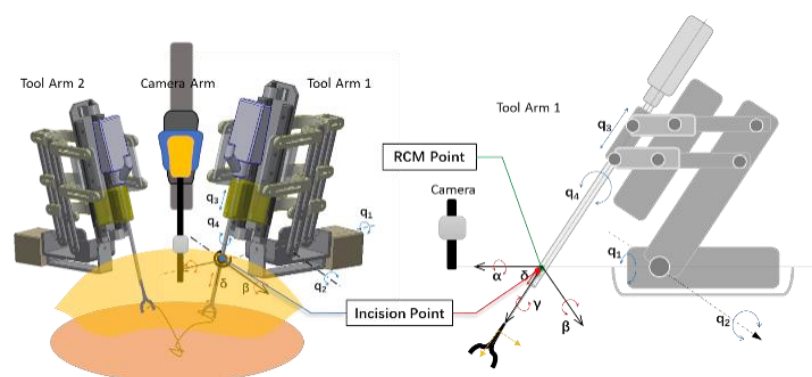

Figure 5. Workspace kinematics of minimally invasive procedures (left); Robotics kinematics to perform minimally invasive procedure (right).

Through this kinematic constraint, we can be sure that the laparoscopic tool shaft will always be declining away from the camera image plane during the procedure as illustrated in Figure 6. Because of the projective geometry, the portion of the shaft near to the camera will appear wider apart. Since the camera view is directed at the surgical site where the tool shaft advance towards, the expected $2 \mathrm{D}$ view will have the shaft linear outline converging towards the center of the view as the shaft decline axially away from the camera optical center.
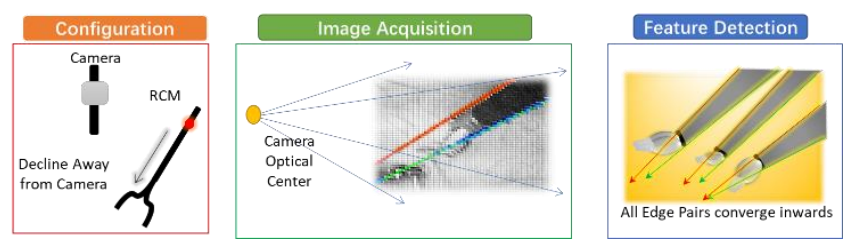

Figure 6. Schematic of the procedural configuration (left); Projective geometry of the image acquisition making sure that the portioin of the shaft nearer to the camera is wider apart (middle); Edge feature pairs converge inwards as a result of the RCM constraint (right).

This kinematic constraint results in a logical condition that is true throughout the video sequence and can be mathematically represented as

$$
{ }^{k} \theta_{E d g e A}-{ }^{k} \theta_{E d g e B}>0,
$$


within a common frame $\mathrm{k}$ of the video sequence where $\theta_{\mathrm{EdgeA}}$ and $\theta_{\mathrm{EdgeB}}$ are the azimuth components of the polar coordinates in the Hough space associated with Edge A and B of the tool shaft, respectively. There is no absolute order to which edge A or B refers to. As long as they are consistently the same edges throughout, the logical condition holds. This constraint is a useful condition for outlier removal as will be discussed in the Results section.

\section{RESULTS}

Actual video data during a robot-assisted surgical procedure is used in this study. There was no prior setup for the tracking. The feature extraction is executed in a mobile workstation Dell Precision 5520; Intel Xeon CPU E3-1505M v6, 3GHz) offline after to procedure. The average computational speed of the geometric feature extraction was $3 \mathrm{~ms}$. This is however within the interest of our application since task modeling and analysis do not need to be carried out on-the-fly.

\subsection{Feature Extraction on Surgical Video Data Set}

To evaluate the feature detection capability, quantitative measurement of the localization error is studied. For the detection of the line edges, all involved candidate points $(N=4171)$ in the voted coordinates are used for measurement of geometrical error which is then used for consistency evaluation. The geometrical error is essentially the shortest distance from an associated candidate point to the extracted line. Figure 7 illustrates the feature extraction of the representative frames in the image sequence.
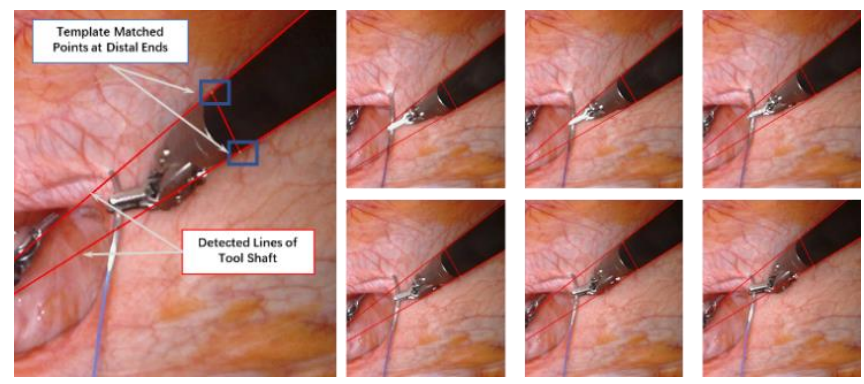

Figure 7. Feature extraction on the video data.

\subsection{Failure Detection}

From the Hough space coordinates we are able to apply the condition in Equation 6 to reject outliers in the feature extraction task. In Figure 8, the three frames can be seen to have opposite sign in the difference of angular incline between the two edges. This results in rejection of the extracted line.

Correspondingly, it can be observed, based on manual visual inspection of the extracted line feature, that three out of four of these failures (Frame 18, 33, 39, 57) were indeed captured by our conditions, with the exception of Frame 33. Validation of the rejected lines are further visually inspected with average errors of the line detection reported in Table 1.

The mean geometric error is essentially the average shortest distance from a candidate point to the determined linear edge in the scene. It can be observed that the is no relation between the accuracy of the detection and the geometric error. The latter is a mere index for consistency of an estimated line from a set of points.
The abnormality in Frame 33 is, nevertheless, apparent in the Hough-space coordinates according to Figure 8. From the plot of the Hough space coordinates sequentially over the frames of the video stream, we could observe a drastic change in neighborhood smoothness in Frame 33. By enforcing continuity and smoothness constraint in the Hough space trajectory, tracking failure like that of Frame 33 could be identified. Figure 9 is a visualization of the detection failure cases.

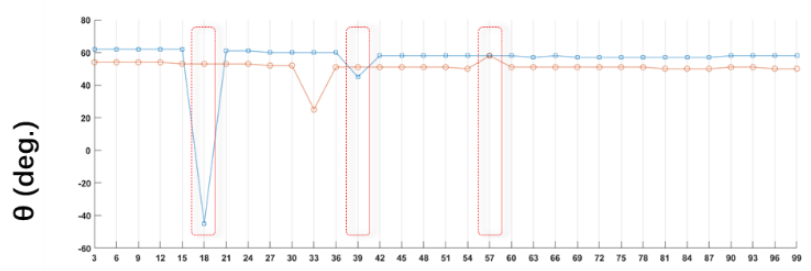

Frame

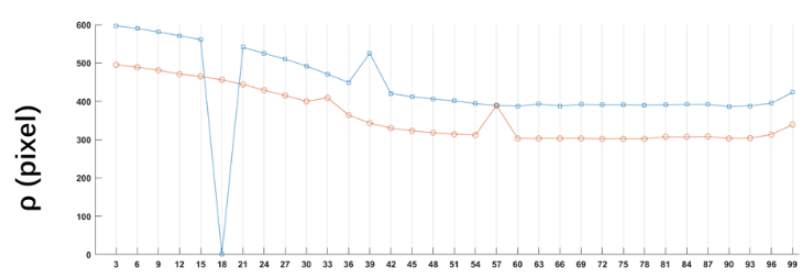

Frame

Figure 8. Hough space coordinates of the feature extraction results. Based on Equation 6 the demarcated frames are rejected downstream of the feature extraction.

Table 1. Rate and Error of Automatic Line Detection

\begin{tabular}{lll}
\hline $\begin{array}{l}\text { Error Detection of 33 } \\
\text { Frames }\end{array}$ & $\begin{array}{l}\text { Mean Geometric } \\
\text { Error(Pixels) }\end{array}$ & $\begin{array}{l}\text { Detection } \\
\text { Rate }^{* *}(\%)\end{array}$ \\
\hline Correctly Detected & 1.35 & 87.9 \\
Correctly Rejected & 1.10 & 9.09 \\
Erroneously Detected & 1.27 & 3.33 \\
Erroneously Rejected & N.A. & 0 \\
\hline & $* *$ Validated through visual inspection
\end{tabular}
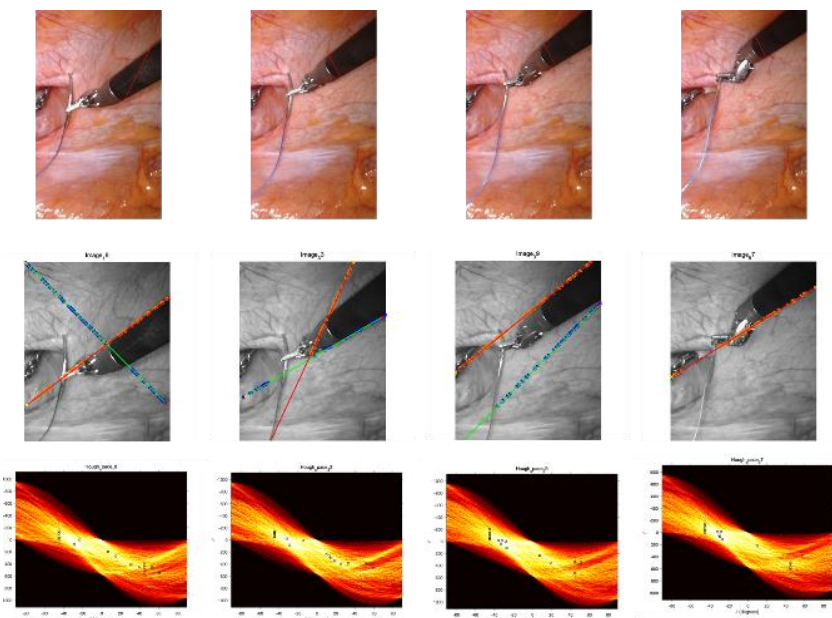

Figure 9. Visualization of detection failure. 
Even though detected edges in Frame 33 does not violate the static frame constraint in Equation (6), the change in the upper edge is substantial in the Hough space compared to its temporal neighboring frames. Any form of sequential filtering that work on the kinematic constraint of continuity would have easily identify the failure in Frame 33. Threshold of the change in trajectory gradient could be specified by the user based on the assumed model of the task or the temporal resolution of the video stream.

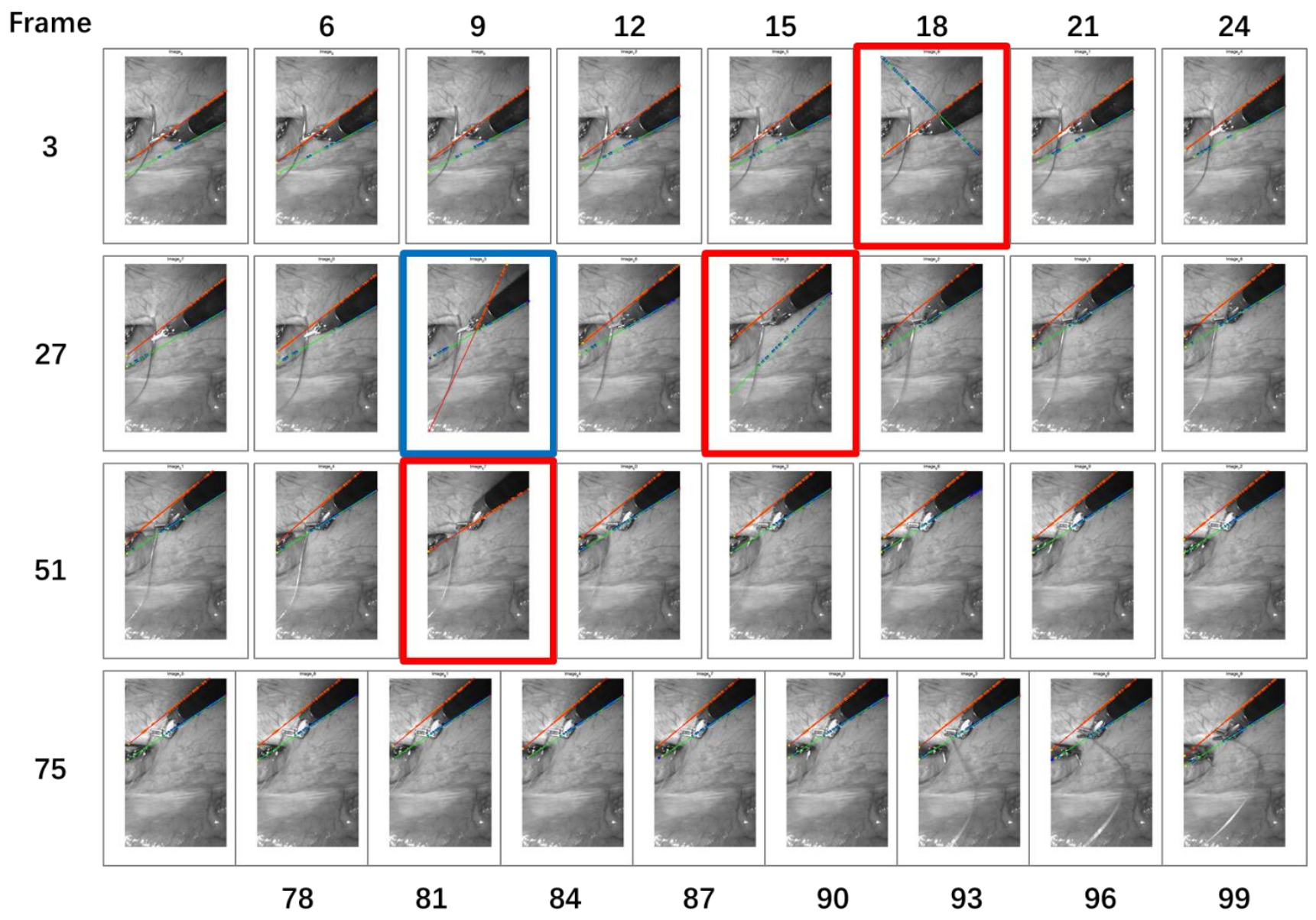

Figure 10. Visual inspection of all 33 frames (3-fame intervals over 99 frames of video segment); Red boarders indicate detected failures; Blue border indicates undetected failure but identified in retrospect through visual inspection.

\section{DISCUSSION}

Without applying pre-processing steps on the images, influence from shadow or sudden motion can affect the tracking. Apart from visually inspecting all the presented 33 frames in the experimental study, specific conditions were also investigated to look at adverse influence on the tracking robustness. Two identified adverse influences include shadow artefacts and sudden motion as seen in Figure 11. The shadow artefacts may result in misdetection of the shaft edges while a drastic sudden change in movement of the tool can affect the accuracy of the template-based matching.

As seen in the right segment of the feature extraction prior to image preprocessing in Figure 11, the existence of the shaft's shadow could lead to erroneous selection of the edge. However, in our application, it is obvious to consider a suitable threshold during the edge detection and binarization to readily remove such undesirable artefact. We can assume that for general surgical
The visual inspection of all 33 frames are shown in Fig. 10. These 33 frames are regular 3-frame intervals of a video segment 99frame sequence. Detected failures are demarcated with red boarders while the blue border of Frame 33 indicates undetected failure but identified in retrospect through visual inspection. 
substantial motion throughout the procedure. There have been some previous studies demonstrating the collaborative use of motion- and template-based visual trackers for trajectory tracking of tools for micromanipulation [41-45].
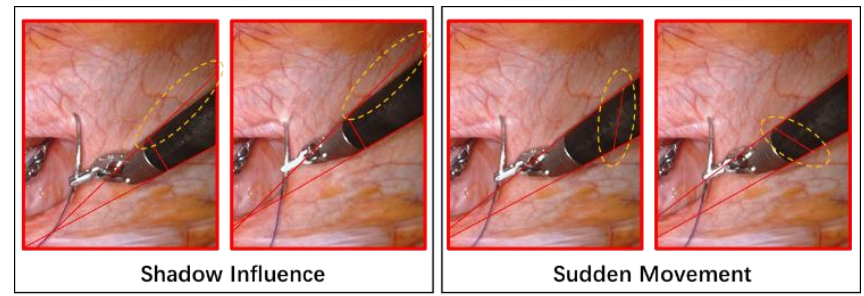

Figure 11. Visualization of adverse influence.

\section{CONCLUSION AND FUTURE WORK}

This paper demonstrated feature extraction that includes a failure detection technique in the Hough space based on the kinematics of minimally invasive procedure. Results and analysis are based on surgical video data of a Robot-assisted minimally invasive procedure showing accurate detection rate of $87.9 \%$. It is expected that the ubiquity of the proposed design of the technology could overcome existing technical bottlenecks in vision-based trajectory tracking and meet the need of increasing demand for data-driven approaches in medical interventional workflow.

Future work will include the development of 2D-3D registration method given the Computer-Aided-Design (CAD) model of the specific tool $[22,23]$ as well as task modeling and analysis based on the extracted trajectory. This extracted information will empower the future of medicine with unprecedented scientific insights and technical breakthrough transforming surgical procedure from a highly competence-reliant operation to a more systematic data-driven workflow. The developed visual tracking approach lay a foundation for surgical task modeling and analysis through video data analysis of robot-assisted minimally invasive procedures.

\section{ACKNOWLEDGMENTS}

This work was supported by the Zhejiang University/University of Illinois at Urbana-Champaign Institute, and Natural Science Foundation of Zhejiang Province (LQ19F030013) led by Principal Supervisor Liangjing Yang. We would also like to express our gratitude to Dr. David Crawford from OSF healthcare St. Francis Medical center in sharing the video segments for his robotassisted minimally invasive procedures.

\section{REFERENCES}

[1] V. Vitiello, S.-L. Lee, T. P. Cundy, and G.-Z. Yang, "Emerging robotic platforms for minimally invasive surgery," IEEE reviews in biomedical engineering, vol. 6, pp. 111-126, 2013.

[2] L. Huffmanm et al., "Robotic Heller myotomy: a safe operation with higher postoperative quality-of-life indices," vol. 142, no. 4, pp. 613-620, 2007.

[3] T. Walther et al., "Pain and quality of life after minimally invasive versus conventional cardiac surgery," vol. 67 , no. 6 , pp. 1643-1647, 1999.

[4] D. Stoyanov, "Surgical vision," Annals of biomedical engineering, vol. 40, no. 2, pp. 332-345, 2012.

[5] M. Hoeckelmann, I. J. Rudas, P. Fiorini, F. Kirchner, and T. Haidegger, "Current capabilities and development potential in surgical robotics," International Journal of Advanced Robotic Systems, vol. 12, no. 5, p. 61, 2015.

[6] K. Cleary and T. M. Peters, "Image-guided interventions: technology review and clinical applications," Annual review of biomedical engineering, vol. 12, pp. 119-142, 2010.

[7] T. Peters and K. Cleary, Image-guided interventions: technology and applications. Springer Science \& Business Media, 2008.

[8] L. Maier-Hein et al., "Surgical data science for nextgeneration interventions," vol. 1, no. 9, p. 691, 2017.

[9] S. S. Vedula and G. D. J. I. S. S. Hager, "Surgical data science: the new knowledge domain," vol. 2, no. 3, pp. 109121, 2017.

[10] T. Yang et al., "Mechanism of a learning robot manipulator for laparoscopic surgical training," in Frontiers of Intelligent Autonomous Systems: Springer Berlin Heidelberg, 2013, pp. 297-308.

[11] S. Tsuda, D. Scott, J. Doyle, and D. B. Jones, "Surgical Skills Training and Simulation," Current Problems in Surgery, vol. 46, no. 4, pp. 271-370, 4// 2009.

[12] D. J. Scott, C. M. Pugh, E. M. Ritter, L. M. Jacobs, C. A. Pellegrini, and A. K. Sachdeva, "New directions in simulation-based surgical education and training: Validation and transfer of surgical skills, use of nonsurgeons as faculty, use of simulation to screen and select surgery residents, and long-term follow-up of learners," Surgery, vol. 149, no. 6, pp. 735-744, 6// 2011.

[13] M. O'Malley et al., "Robotics as a Tool for Training and Assessment of Surgical Skill," in Computational Surgery and Dual Training, M. Garbey, B. L. Bass, S. Berceli, C. Collet, and P. Cerveri, Eds.: Springer New York, 2014, pp. 365-375.

[14]B. Chebbi, D. Lazaroff, F. Bogsany, P. X. Liu, L. Niy, and M. Rossi, "Design and implementation of a collaborative virtual haptic surgical training system," in Mechatronics and Automation, 2005 IEEE International Conference, 2005, vol. 1, pp. 315-320 Vol. 1.

[15] A. J. Chowriappa et al., "Development and validation of a composite scoring system for robot-assisted surgical training - the Robotic Skills Assessment Score," vol. 185, no. 2, pp. 561-569, 2013.

[16] M. Uemura et al., "Procedural surgical skill assessment in laparoscopic training environments," vol. 11, no. 4, pp. 543552, 2016.

[17] K. Ahmed, D. Miskovic, A. Darzi, T. Athanasiou, and G. B. J. T. A. J. o. S. Hanna, "Observational tools for assessment of procedural skills: a systematic review," vol. 202, no. 4, pp. 469-480. e6, 2011.

[18] J. Stoll, H. Ren, and P. E. Dupont, "Passive Markers for Tracking Surgical Instruments in Real-Time 3-D Ultrasound Imaging," Medical Imaging, IEEE Transactions on, vol. 31, no. 3, pp. 563-575, 2012.

[19] H. Ren and P. Kazanzides, "Investigation of attitude tracking using an integrated inertial and magnetic navigation system for hand-held surgical instruments," Mechatronics, IEEE/ASME Transactions on, vol. 17, no. 2, pp. 210-217, 2012.

[20] J. Mung, F. Vignon, and A. Jain, "A non-disruptive technology for robust 3D tool tracking for ultrasound-guided 
interventions," in Medical Image Computing and ComputerAssisted Intervention-MICCAI 2011: Springer, 2011, pp. 153-160.

[21] K. Mori et al., "Hybrid bronchoscope tracking using a magnetic tracking sensor and image registration," in Medical Image Computing and Computer-Assisted InterventionMICCAI 2005: Springer, 2005, pp. 543-550.

[22] P. Markelj, D. Tomaževič, B. Likar, and F. J. M. i. a. Pernuš, "A review of 3D/2D registration methods for image-guided interventions," vol. 16, no. 3, pp. 642-661, 2012.

[23] R. Sandhu, S. Dambreville, A. Yezzi, and A. Tannenbaum, "Non-rigid 2D-3D pose estimation and 2D image segmentation," in 2009 IEEE Conference on Computer Vision and Pattern Recognition, 2009, pp. 786-793: IEEE.

[24] J. Wang et al., "Augmented Reality Navigation With Automatic Marker-Free Image Registration Using 3-D Image Overlay for Dental Surgery," Ieee Transactions on Biomedical Engineering, vol. 61, no. 4, pp. 1295-1304, APR 20142014.

[25] J. Wang et al., "Real-time computer-generated integral imaging and 3D image calibration for augmented reality surgical navigation," Computerized Medical Imaging and Graphics, vol. 40, no. 0, pp. 147-159, 3// 2015.

[26] J. Wang, H. Suenaga, L. Yang, E. Kobayashi, and I. Sakuma, "Video see-through augmented reality for oral and maxillofacial surgery," The International Journal of Medical Robotics and Computer Assisted Surgery, 20162016.

[27] D. H. Ballard, "Generalizing the Hough transform to detect arbitrary shapes," Pattern recognition, vol. 13, no. 2, pp. 111-122, 1981.

[28] J. Canny, "A computational approach to edge detection," IEEE Transactions on pattern analysis and machine intelligence, no. 6, pp. 679-698, 1986.

[29] O. G. Grasa, J. Civera, and J. Montiel, "EKF monocular SLAM with relocalization for laparoscopic sequences," in Robotics and Automation (ICRA), 2011 IEEE International Conference on, 2011, pp. 4816-4821: IEEE.

[30] J. Civera, O. G. Grasa, A. J. Davison, and J. Montiel, "1point RANSAC for EKF-based structure from motion," in Intelligent Robots and Systems, 2009. IROS 2009. IEEE/RSJ International Conference on, 2009, pp. 3498-3504: IEEE.

[31] O. G. Grasa, J. Civera, A. Guemes, V. Munoz, and J. Montiel, "EKF monocular SLAM 3D modeling, measuring and augmented reality from endoscope image sequences," in Medical Image Computing and Computer-Assisted Intervention (MICCAI), 2009, vol. 2.

[32] L. Yang, I. Paranawithana, K. Youcef-Toumi, and U. Tan, "Self-initialization and recovery for uninterrupted tracking in vision-guided micromanipulation," in 2017 IEEE/RSJ International Conference on Intelligent Robots and Systems (IROS), 2017, pp. 1127-1133.

[33] L. Yang et al., "Towards scene adaptive image correspondence for placental vasculature mosaic in computer assisted fetoscopic procedures," The International Journal of Medical Robotics and Computer Assisted Surgery, vol. 12, no. 3, pp. 375--386, oct 2015.
[34] L. Yang et al., "Ultrasound image-based endoscope localization for minimally invasive fetoscopic surgery," in Proceedings of the Annual International Conference of the IEEE Engineering in Medicine and Biology Society, EMBS, 2013, pp. 1410-1413.

[35] Z. Zhang, "Flexible camera calibration by viewing a plane from unknown orientations," in Computer Vision, 1999. The Proceedings of the Seventh IEEE International Conference on, 1999, vol. 1, pp. 666-673: IEEE.

[36] C.-B. Chng, B. Duan, and C.-K. Chui, "Modeling and simulation of a Remote Center of Motion mechanism," in Region 10 Conference (TENCON), 2016 IEEE, 2016, pp. 1755-1758: IEEE.

[37] L. Yang, C. B. Chng, C. K. Chui, and D. P. C. Lau, "Modelbased design analysis for programmable remote center of motion in minimally invasive surgery," in 2010 IEEE Conference on Robotics, Automation and Mechatronics, RAM 2010, 2010, pp. 84-89.

[38] L. Matthews, T. Ishikawa, and S. Baker, "The template update problem," IEEE Transactions on Pattern Analysis and Machine Intelligence, vol. 26, no. 6, pp. 810-815, 2004.

[39] A. Malti, T. Collins, and A. Bartoli, "Template-Based Deformable Shape-from Motion from Registered Laparoscopic Images," Medical Image Understanding and Analysis (MIUA'11), 2011.

[40] Z. Kalal, K. Mikolajczyk, J. J. I. t. o. p. a. Matas, and m. intelligence, "Tracking-learning-detection," vol. 34, no. 7, pp. 1409-1422, 2012.

[41]L. Yang, I. Paranawithana, K. Youcef-Toumi, and U.-X. Tan, "Self-Initialization and Recovery for Uninterrupted Tracking in Vision-Guided Micromanipulation," presented at the IEEE International Conference on Intelligent Robots and Systems, IROS'2017, Vancouver, Canada, 2017.

[42] I. Paranawithana, L. Yang, Z. Chen, K. Youcef-Toumi, and U.-X. Tan, "Scene-Adaptive Fusion of Visual and Motion Tracking for Vision-Guided Micromanipulation in Plant Cells," presented at the 2018 IEEE International Conference on Automation Science and Engineering (CASE), Munich, Germany, 2018.

[43] L. Yang, K. Youcef-Toumi, and U.-X. Tan, "Detect-FocusTrack-Servo (DFTS): A Vision-Based Workflow Algorithm for Robotic Image-Guided Micromanipulation," presented at the IEEE International Conference on Robotics and Automation, ICRA'2017, Singapore, Singapore, 2017 (in press).

[44] L. Yang, K. Youcef-Toumi, and U.-X. Tan, "Towards automatic robot-assisted microscopy: An uncalibrated approach for robotic vision-guided micromanipulation," in Intelligent Robots and Systems (IROS), 2016 IEEE/RSJ International Conference on, 2016, pp. 5527-5532: IEEE.

[45]L. Yang, I. Paranawithana, K. Youcef-Toumi, and U.-X. Tan, "Automatic Vision-Guided Micromanipulation for Versatile Deployment and Portable Setup," IEEE Transactions on Automation Science and Engineering, vol. (Accepted), 2017. 